\title{
Investigating the Planning and Translating Processes in Foreign Language Reading-to-Write
}

\author{
Pucheng Wang \\ School of Applied Foreign Languages, Zhejiang International Studies University, Hangzhou, China \\ Email:p.wang@zisu.edu.cn
}

How to cite this paper: Wang, P. C. (2022). Investigating the Planning and Translating Processes in Foreign Language Reading-toWrite. Advances in Literary Study, 10, 141149.

https://doi.org/10.4236/als.2022.102011

Received: February 13, 2022

Accepted: March 5, 2022

Published: March 8, 2022

Copyright (อ 2022 by author(s) and Scientific Research Publishing Inc. This work is licensed under the Creative Commons Attribution International License (CC BY 4.0).

http://creativecommons.org/licenses/by/4.0/

\begin{abstract}
This study looked at how English as a Foreign Language (EFL) writers formulated macro and micro writing plans, as well as how they translated abstract ideas into concrete linguistic forms while completing a reading-to-write task. Results showed that most of the participants engaged in planning and translating processes during task completion. They appeared to focus on planning the text's content, with little thought given to the intended readers or the piece's genre and style. There is also evidence that the participants used micro-planning processes when planning at the sentence and paragraph levels, with the processes of selecting and connecting being used frequently to aid the micro-planning process. The results of the micro-planning process may have been stored in the minds of the participants in the form of abstract thoughts, which were then likely translated into verbal forms.
\end{abstract}

\section{Keywords}

Second/Foreign Language Writing, Integrated Writing Tasks, Macro-Planning, Micro-Planning, Translating

\section{Introduction}

The use of integrated writing tasks in second/foreign language (L2/FL) teaching and assessment has grown in popularity over the past four decades (Gebril \& Plakans, 2013; Golparvar \& Khafi, 2021; Shi, 2004; Spivey, 1984, 1997; Wang \& Zhang, 2021; Weigle \& Parker, 2012). It is generally believed that writing is thought to be unlikely to be done independently of other language skills; rather, it relies on gathering information from external sources either through reading, listening, or both (Esmaeili, 2002; Hinkel, 2006; Hirvela, 2004). Reading-to-write tasks require writers to use both their reading and writing skills. One important process that has been extensively examined in this type of task is the process of 
planning. It is argued that, when completing reading-to-write tasks, the planning process requires more of the writers' reading abilities due to the inclusion of source materials, making the process more complex, as contrasted to planning for an independent writing task (Chan, 2013).

To illustrate the different purposes of planning activities, Field (2004) stated that there are two distinct types of planning processes: macro-planning and $\mathrm{mi}-$ cro-planning. Macro-planning is a process in which writers plan for the writing goals and content, as well as identify major constraints of the task such as the target readership, genre, and the level of formality required (Field, 2004; Shaw \& Weir, 2007). Unskilled writers, according to Scardamalia and Bereiter (1987), did not appear to use the macro-planning process because they used a knowledge-telling approach to writing, retrieving and listing ideas from their longterm memory in a rather linear manner; skilled writers, on the other hand, tended to take a knowledge-transforming approach to writing, putting a lot of effort into macro-planning to guide their writing.

As Field (2004) argued, planning processes can take place not only at the macro-level, but also at the micro-level, i.e., at the sentence and paragraph levels. During the micro-planning process, writers plan the text that is about to be produced. At the paragraph level, writers plan the goals, content, and structure of a paragraph, possibly with constant reference to the macro-plans established earlier (for example, the overall writing goal, genre, and level of formality) as well as the text written thus far. At the sentence level, writers plan the structure and content of an upcoming sentence. Micro-plans, rather than macro-plans, are thought to be used in the actual text production process (Field, 2004).

The results of the micro-planning process are stored in the minds of writers as specific goals at the paragraph and sentence levels, which then serve as the foundations for the translating process, during which writers' abstract ideas are transformed into concrete linguistic forms. Shaw and Weir (2007: p. 39) claimed that it is through the translating process that "the writer moves from an internal 'private' representation, which is abstract and only understood by him or her, to its expression in the 'public' shared code of language". They also argued that the language translated needs to be not only lexically and syntactically correct but also functionally appropriate.

Planning and translation processes are critical when writers make writing plans at both the macro and micro levels, and then carry out these plans to produce text. However, compared to other processes involved in writing, they may be more difficult to be investigated reliably as writers tend to be less aware of their use (particularly the use of micro-planning and translating processes). The majority of studies that attempted to investigate writers' planning and translating processes looked at the processes by examining writers' offline written products; Only a few studies looked at writers' online planning and translating activities.

Therefore, there is a need to improve our understanding of how L2 or FL 
writers plan and translate while reading and writing, particularly from an online-investigation perspective, in order to inform language instructors and learners about the nature of these processes and facilitate their teaching and learning. The present study used an eye-tracking technique and a stimulated recall method to examine $16 \mathrm{EFL}$ writers' planning and translating processes in responding to a reading-to-write task, with the goal of triangulating data from multiple sources and learning more about writers' reading-to-write process in an EFL context. Two research questions were proposed to look into the participants' planning and translating processes:

1) How do EFL writers make macro writing plans as they read and write? (RQ1)

2) How do EFL writers make micro writing plans and translate ideas into linguistic forms as they read and write? (RQ2)

\section{Methods}

\subsection{Participants}

This study involved 16 participants. They were all native Chinese students studying at a UK university. They ranged in age from 21 to 28 years old (Mode=23; Mean = 22.6; $S D=1.66)$, with 11 of them being female (69\%) and 5 being male (31\%). Before the data was collected, all of the participants had completed the International English Language Testing System (IELTS) test. Table 1 displays their results. These participants had English proficiency levels ranging from B2 to $\mathrm{C} 1$ according to the Common European Framework of Reference for Languages (CEFR).

\subsection{Instrument and Data Collection}

The participants' eye movements were recorded using a Tobii TX300 eye-tracker as they responded to the stimulus onscreen, which was a sample reading-towrite task from the Test for Business English Majors-Band 8 (TBEM-8, developed and administered in China). A task instruction and five source materials about Steve Jobs' resignation from Apple make up the task prompt. The task was presented on the eye-tracker screen in two parts: the instruction and three source texts were presented on the left, and the other two source materials, as well as the answer sheet box (where the participants input the text), were presented on the right.

Table 1. Participants' IELTS test scores.

\begin{tabular}{ccccccc}
\hline $\begin{array}{c}\text { IELTS/IELTS } \\
\text { components }\end{array}$ & Mean & Median & Mode & $\begin{array}{c}\text { Standard } \\
\text { Deviation }\end{array}$ & Minimum & Maximum \\
\hline Overall & 7.16 & 7.00 & 7.50 & 0.35 & 6.50 & 7.50 \\
Reading & 8.00 & 8.00 & 8.50 & 0.58 & 7.00 & 9.00 \\
Writing & 6.25 & 6.00 & 6.00 & 0.55 & 5.50 & 7.00 \\
\hline
\end{tabular}


During the data collection, the participants were first performing the reading-to-write task with their eye movements being recorded by the eye-tracker. After they had completed the task, they were then asked to verbalise their thoughts during the task completion using the eye traces recorded by the eyetracker as stimuli for retrospection. The stimulated recall session was conducted in Mandarin Chinese and audio and video recordings were taken for later data analysis.

\subsection{Analysis}

The participants' verbal protocols were first transcribed by the researcher (a native Chinese speaker) based on the audio and video recordings of the stimulated recall session. The transcriptions were then coded to identify participants' planning and translating processes throughout the task completion, and the number of instances of each particular type of these processes (macro-planning, micro-planning and translating) at various stages of task completion (before writing, during writing and after writing) was calculated. Additionally, excerpts from participants' verbal protocols were shown to demonstrate how they used these processes to complete the reading-to-write task. Last, it should be noted that as the combined use of eye-tracking and stimulated recall methods is quite timeconsuming and labour-intensive, a relatively small number of participants were involved in this study, and thus interpreting the results too broadly would be risky, and any conclusions drawn should be seen as tentative.

\section{Results and Discussion}

\subsection{Macro-Planning Process (RQ1)}

During the macro-planning process, writers plan for writing goals and content, and identify major constraints of a task such as genre and the level of formality required, the target readership, etc., on the basis of their representation of task (Field, 2004; Shaw \& Weir, 2007).

Twelve participants reported that they used macro-planning processes (27 instances) while completing the reading-to-write task. Almost all of these instances occurred before the participants started to compose and they were mainly concerned with goal setting and consideration of content, for example, Participant 5 said that she "divided the content requirements in the instructions into several parts, and planned the paragraphs to be written according to the focus in each part...", and Participant 6 reported that "when I was reading (the source materials), I already began to think about what content I should wrote, because I thought that I did not have to write everything in the materials, for example, I decided not to write about Jobs' death". No instances of consideration of the genre and target readership were found in the protocols, which indicates that the participants may not have been aware of the importance of these two aspects in successful task completion.

It should be noted that the macro-planning process employed when complet- 
ing reading-to-write tasks requires more of participants' reading abilities because of the inclusion of source materials, making this process more complex, as compared to macro-planning in completing independent writing tasks. Many participants were found building connections between source materials and macro-plans for their writing, for example, Participant 11 reported that:

I found that the most important thing was to integrate information in these source materials to my writing, and it was not necessary to include many of my own opinions, so I reread the first three materials and categorised them to decide in which paragraph of the essay their information can be put into.

Also, participants were found referring to the task instructions when making macro-plans before writing, for instance, Participant 10 said that he started to make plans for writing after he "understood the content and structure of the source materials" and "referred constantly to the content requirements in the instructions", proving that the process of macro-planning can be influenced by the constraints of the task (Grabe \& Kaplan, 1996; Shaw \& Weir, 2007).

\subsection{Micro-Planning and Translating Process (RQ2)}

It is believed that planning may also take place at the micro-level, i.e., at the sentence and paragraph level (Field, 2004; Shaw \& Weir, 2007), during which writers plan for the goal, content and structure of a particular paragraph or an upcoming sentence, possibly with constant reference back to the macro-plans established earlier as well as the text produced so far.

164 instances of micro-planning process were found in the 16 participants' protocols, the majority of which occurred during writing. At the paragraph level (62 instances), all the participants reported that they engaged in planning for either the content or the overall structure of an upcoming paragraph. For example, Participant 4, when monitoring the progress of task completion, said "At this moment, based on what I had just read, I thought I should add another paragraph here talking about the current status, analysing the impact of Jobs' resignation on Apple company", and Participant 1 stated that " $I$ was going to include two aspects of information in this paragraph, at this moment I was wondering which aspect I should write first, later I decided to first write about the impact on the company within itself".

At the sentence level (102 instances), participants were also found to plan for the content and structure of an upcoming sentence, for example, Participant 8 recalled that " $I$ was writing the topic sentence here, and I was thinking about using which sentence to state the topic...I went to the readings to find possible material, but later I decided to write it myself", and Participant 6 reported on how he went about structuring a sentence, "I was thinking about the structure of this sentence, should I write a simple sentence, or should I write a complex sentence with a relative clause...".

It is worth noting that, the participants were found often going back to read the instructions, source materials or the text that had been produced when they 
were micro-planning at both the sentence and paragraph level. First, for example, Participant 8 stated that she went back to reread the instructions when she finished writing the first paragraph of her essay, "I was looking at the instructions and thinking about what to write in the next paragraph"; the reason for this may be that the participant did not plan well at the macro-level, and so she may have needed to refer back to the instructions to create a clearer representation of the task and redo macro-planning before she continued to plan what content she was going to write in the next paragraph.

Second, when the participants reread the source materials while microplanning, they were either selecting ideas from the materials to connect with their own knowledge to generate new meaning, or just choosing information they may need for their writing, and these processes of selecting and connecting may ultimately generate a micro-plan for the next paragraph or sentence. For instance, Participant 3 recalled that "I had finished writing my first point of view in this part, and then I reread the source materials and the text I had written, and began to think about how to write the second point of view", and Participant 1, when writing a sentence in the third paragraph of her essay, said:

I was thinking about how to write his traits, what words I could use to describe him, and then I went to source material to look for any possible words. I read through almost all the key words that might be useful in the source materials, and now I finally came up with some words in my mind, so I began to write this sentence.

Last, the participants were also found frequently going back to reread the text that they had written, either before starting to write a new sentence or in the middle of producing part of an existing one, for example, Participant 10 recalled that "I was going to write some comments, but it seemed to me that I had not fully described this incident in that sentence, so I went back to read what I had written and the source materials, and decided to add some information into the sentence". This is natural that, as the participants went on writing, the evolving textual output became part of the context that they had to consider in order to drive further planning for the content to be produced.

The output of micro-planning was stored in the participants' minds in the form of goals at the sentence and paragraph levels, which then became the bases of their translating process, during which the abstract ideas were transcribed into concrete linguistic forms. As discussed earlier, it is at this critical point that "the writer moves from an internal 'private' representation, which is abstract and only understood by him or her, to its expression in the 'public' shared code of language" (Shaw \& Weir, 2007: p. 39). The participants reported 33 instances of the process of translating, whose number was very likely to be underestimated, because translating may not be adequately reported as the participants tended to be less aware of the use of it due to its automatized nature, although it is an important process when producing the actual text during writing (Field, 2004); Also, the limitation of stimulated recall methodology may be another factor that 
hinders these participants' reflection on the translating process.

By looking at the limited instances of translating process, together with the participants' eye-traces in the eye-movement recordings, we could see that this process was rather complicated and highly demanding in terms of cognitive processing in the context of L2 writing. This may because it is at this point that L2 writers face critical problems regarding the translation of abstract ideas for which they may not possess necessary language resources. In other words, their knowledge of, for example, vocabulary and grammar of L2 may not be adequate to represent the ideas stored in the micro-plans. For example, Participant 12 stated in her protocol that, "I was thinking about how to write this sentence, I already had an idea of what I was going to write, but I kept thinking about the language issues".

Problems related to language resources appeared to exert additional cognitive demand on the participants' translation process, during which they were very likely turning to the source materials provided in the task to seek both lexical and syntactical support, which was evidenced in their stimulated recalls and eye-tracking traces. For example, participants frequently, especially in the middle of a sentence production, referred to source materials for extra support, which was often at a very detailed level, for example, looking for specific words or sentence structures, or even either copying, translating or paraphrasing the information in the input texts. The high cognitive demand on the translating process may, in turn, have hindered the execution of other processes such as connecting and organising, and thus have impacted on the quality of the final written product.

\section{Conclusion}

This study investigated EFL writers' planning and translating processes in responding to a source-based reading-to-write task. Findings from the participants' verbal protocols reveal that, first, as they read and write to complete the task, most of them used macro-planning processes; they seemed to focus on the planning of the content of the text, while little effort was made to consider the target readership, or the genre and style of the piece; writers' macro-planning process not only involved gathering of ideas, but also building connections between source materials and plans for writing to determine what and how the text was to be written to successfully complete the task.

Second, there is evidence of the 16 participants using micro-planning process when they conducted planning and organising at both the sentence and paragraph levels, during which the processes of text interpretation, selecting and connecting were also frequently employed to facilitate the process of microplanning; the output of micro-planning process may be, to a certain degree, stored in the participants' mind in the form of abstract ideas, which were then likely to be translated into linguistic forms.

The study's major outcome is a better understanding of how EFL writers make 
writing plans at both the macro and micro levels, as well as how the translating process impacts on the reading-to-write activities, with which EFL instructors may improve their teaching plans to teach these implicit but essential cognitive processes in reading-to-write. There are also some methodological implications that the combined use of eye-tracking and stimulated recall might yield rich data from participants and offer a strong foundation on which inferences about their cognitive processing when reading and writing in a foreign language could be derived.

\section{Conflicts of Interest}

The author declares no conflicts of interest regarding the publication of this paper.

\section{References}

Chan, S. H. C. (2013). Establishing the Validity of Reading-into-Writing Test Tasks for the UK Academic Context. Unpublished PhD Thesis, The University of Bedfordshire.

Esmaeili, H. (2002). Integrated Reading and Writing Tasks and ESL Students' Reading and Writing Performance in an English Language Test. The Canadian Modern Language Review, 58, 599-622. https://doi.org/10.3138/cmlr.58.4.599

Field, J. (2004). Psycholinguistics: The Key Concepts. Routledge. https://doi.org/10.4324/9780203506929

Gebril, A., \& Plakans, L. (2013). Toward a Transparent Construct of Reading-to-Write Tasks: The Interface between Discourse Features and Proficiency. Language Assessment Quarterly, 10, 9-27. https://doi.org/10.1080/15434303.2011.642040

Golparvar, S. E., \& Khafi, A. (2021). The Role of L2 Writing Self-Efficacy in Integrated Writing Strategy Use and Performance. Assessing Writing, 47, 1-15. https://doi.org/10.1016/j.asw.2020.100504

Grabe, W., \& Kaplan, F. L. (1996). Theory and Practice of Writing: An Applied Linguistic Perspective. Longman.

Hinkel, E. (2006). Current Perspectives on Teaching the Four Skills. TESOL Quarterly, 40, 109-131. https://doi.org/10.2307/40264513

Hirvela, A. (2004). Connecting Reading and Writing in Second Language Writing Instruction. The University of Michigan Press. https://doi.org/10.3998/mpub.23736

Scardamalia, M., \& Bereiter, C. (1987). Knowledge Telling and Knowledge Transforming in Written Composition. In S. Rosenberg (Ed.), Advances in Applied Psycholinguistics, Volume 2: Reading, Writing and Language Learning (pp. 142-175). Cambridge University Press.

Shaw, S., \& Weir, C. J. (2007). Examining Writing: Research and Practice in Assessing Second Language Writing, Studies in Language Testing 26. UCLES/Cambridge University Press.

Shi, L. (2004). Textual Borrowing in Second-Language Writing. Written Communication, 21, 171-200. https://doi.org/10.1177/0741088303262846

Spivey, N. N. (1984). Discourse Synthesis: Constructing Texts in Reading and Writing. Outstanding Dissertation Monograph, International Reading Association.

Spivey, N. N. (1997). The Constructivist Metaphor: Reading, Writing and the Making of Meaning. Academic Press. https://doi.org/10.2307/358470 
Wang, P. C., \& Zhang, Z. G. (2021). Constructive Processes in Completing Readingto-Write Tasks: Selecting, Organising and Connecting. Open Journal of Modern Linguistics, 11, 919-930. https://doi.org/10.4236/ojml.2021.116071

Weigle, S. C., \& Parker, K. (2012). Source Text Borrowing in an Integrated Reading/Writing Assessment. Journal of Second Language Writing, 21, 118-133.

https://doi.org/10.1016/j.jslw.2012.03.004 